\title{
BIFURCATION AND STABILITY OF POSITIVE SOLUTIONS OF A TWO-POINT BOUNDARY VALUE PROBLEM
}

\author{
SHIN-HWA WANG and NICHOLAS D. KAZARINOFF ${ }^{\dagger}$
}

(Received 6 June 1989; revised 18 August 1990)

Communicated by E. N. Dancer

\begin{abstract}
We consider the existence of multiple positive solutions of a nonlinear two-point boundary value problem by modifying a "time map" technique introduced by J. Smoller and A. Wasserman. We count the number of positive solutions and find their Conley indices and thus determine their stabilities.
\end{abstract}

1991 Mathematics subject classification (Amer. Math. Soc.): 58 F 14, 34 B 15.

Keywords and phrases: bifurcation, stability, positive solution, two-point boundary value problem, and Conley index.

\section{Introduction}

In this paper, we consider the local bifurcation of positive solutions of the two-point boundary value problem

$$
\begin{gathered}
u^{\prime \prime}+f(u)=0, \quad-L<x<L, \\
u(-L)=u(L)=0,
\end{gathered}
$$

where $2 L>0$, the interval length, is a real bifurcation parameter. Throughout this paper, we assume that $f \in C^{2}[0,1]$ and satisfies

$$
f(x)>0 \text { on }(0,1), f(1)=0,
$$

(C) 1992 Australian Mathematical Society 0263-6115/92\$A2.00+0.00

$\dagger$ Deceased. Nicholas D. Kazarinoff died in November 1991. He was a fine mathematician and a respected friend.

This work was partially supported by the National Science Council of Republic of China. 
and there exists a $\delta>0$ such that $f^{\prime}(x) \leq 0$ on $(1-\delta, 1)$. We obtain local bifurcation diagrams of positive solutions $u$ of (1.1) satisfying

$$
0<\|u\|_{\infty}<1 \text {; }
$$

that is, we count the exact number of positive solutions in the order interval $(0,1)$. Notice that if $f(0)=0$, then $u \equiv 0$ is a solution, and the strong maximum principle shows $f\left(u_{\max }\right)>0$ [2, Lemma 6.2]. We are interested in nonconstant positive solutions.

Equation (1.1) with $f$ satisfying (1.2) is related to the classic Kolmogoroff equation $u_{t}=\frac{1}{2} u_{x x}+f(u)$ which arose in the context of a genetics model for the spread of an advantageous gene through a population [4]. In addition to (1.2), the function $f$ in the classic Kolmogoroff equation satisfies $f(0)=0$ and $f^{\prime}(0)>f^{\prime}(u)$ for $0<u \leq 1[4,8]$. The research in this paper is motivated by papers of T. I. Seidman [10], D. G. de Figueiredo [6], and $H$. Berestycki [3] in which uniqueness results for positive solution of the Dirichlet problem

$$
\begin{gathered}
\Delta u+\lambda f(u)=0 \quad \text { in } \Omega\left(\Omega \text { is a smooth bounded domain in } \mathbb{R}^{m}(m \geq 1)\right) . \\
u=0 \quad \text { on } \partial \Omega,
\end{gathered}
$$

satisfying (1.3) were obtained for some particular $f$ 's. In addition to (1.2), they assumed that $f$ satisfies

$$
f \in C \text { and is nonincreasing on }(0,1) \text { ( T. I. Seidman [10]) }
$$

or

$$
f \in C^{2} \text { and } f^{\prime \prime}(x)<0 \text { on }(0,1)
$$

(D. G. de Figueiredo [6] and H. Berestycki [3]). Therefore, it is of interest to study the multiplicity problem if $f$ is neither nonincreasing nor concave. Among the papers related to this question are [1] by Ambrosetti, [5] by Dancer, and [7] by Gelfand.

We note that if we make the change of variable $y=x / L$, then (1.1) becomes

$$
\begin{gathered}
u_{y y}+L^{2} f(u)=0, \quad|y|<1, \\
u( \pm 1)=0,
\end{gathered}
$$

so that if $\lambda=L^{2}$, we obtain a problem of the familiar type. We prefer, however, to consider the equation (1.1) because as we shall see, its solutions can be given a nice geometrical interpretation (see [11, p. 185]).

We study (1.1) through an approach due to J. Smoller and A. Wasserman [12] who studied (1.1) by the technique of "time map" $T(\alpha)$ to count the exact number of solutions of (1.1) for $f$ a cubic polynomial. 


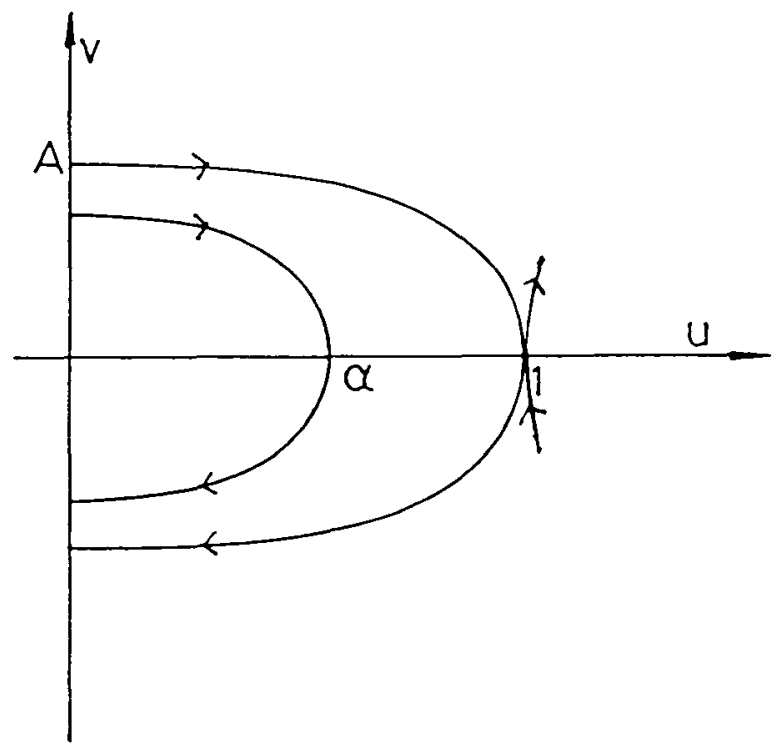

FIGURE 1

As in [12], we rewrite (1.1) as a first order system

$$
u^{\prime}=v, \quad v^{\prime}=-f(u), \quad|x|<L,
$$

and we consider the phase plane for (1.8) locally illustrated in Figure 1.

It is clear that positive solutions of (1.1) satisfying (1.3) correspond to those orbits of (1.8) which "begin" on the interval $(0, A)\left(A>0, A^{2} / 2=\right.$ $F(1)$, where $\left.F(s) \equiv \int_{0}^{s} f(u) d u\right)$ on the $v$-axis, and "end" on the $v$-axis, and take "time" (parameter length) $2 L$ to make the journey. Note that (1.8) is a reversible system; see $\mathbf{M}$. B. Sevryuk [9]. Then, as in [12], we define the time map

$$
T(\alpha)=2^{-1 / 2} \int_{0}^{\alpha}(F(\alpha)-F(u))^{-1 / 2} d u \quad 0<\alpha<1 .
$$

Notice that, solutions of (1.8) correspond to curves for which $T(\alpha)=L$. This led us to investigate the shape of the graph of $T$ (see [11, pp. 186187]).

REMARK. The equation $T(\alpha)=L$ has at most a finite number of solutions $\alpha$ for generic $L$ or generic $f$ which implies (1.1) has finitely many positive solutions satisfying (1.3) with $f$ satisfying (1.2) [13, Proposition 5].

The two estimates (2.3) and (2.5) in [12], we write as (1.10) and (1.14) below:

$$
T^{\prime}(\alpha)=2^{-3 / 2} \int_{0}^{\alpha} \frac{\theta(\alpha)-\theta(u)}{(\Delta F)^{3 / 2}} \frac{d u}{\alpha},
$$


where $\Delta F=F(\alpha)-F(u)$, and

$$
\theta(x)=2 F(x)-x f(x)
$$

Hence, (1.11) gives

$$
\theta^{\prime}(x)=f(x)-x f^{\prime}(x)
$$

and

$$
\theta^{\prime \prime}(x)=-x f^{\prime \prime}(x)
$$

Also, we have

$$
T^{\prime \prime}(\alpha)+\frac{2}{\alpha} T^{\prime}(\alpha)>\frac{2^{-3 / 2}}{\alpha^{2}} \int_{0}^{\alpha}(\Delta F)^{-3 / 2}(\phi(\alpha)-\phi(u)) d u,
$$

where $\phi(x)=x \theta^{\prime}(x)-\theta(x)$.

REMARK. No analysis on $\theta^{\prime \prime}$ was used in [12]; but it is of importance in our analysis (also see $[14,15]$ ).

\section{Main Result}

TheOREM. Suppose, in addition to (1.2),

$$
f(0)=0 \text {, }
$$

and

(2.2) there exists a number $a \in(0,1)$, which is the first zero of $f^{\prime \prime}$ in $(0,1)$ and suppose that $f^{\prime \prime}(x)>0$ in $(0, a)$ and $f^{\prime \prime}(x) \leq 0$ in $(a, 1)$.

Then $T$ defined by (1.9) satisfies

$T$ has exactly one critical point, a minimum, at $\alpha_{0}$ for some $\alpha_{0}$ in $(0,1)$,

$$
T(0)= \begin{cases}\frac{\pi}{2} f^{\prime}(0)^{-1 / 2} & \text { if } f^{\prime}(0)>0 \\ \infty & \text { if } f^{\prime}(0)=0\end{cases}
$$

and

$$
T(1)=\infty
$$

Moreover, $T(\alpha)$ is strictly decreasing in $\left(0, \alpha_{0}\right)$ and $\alpha T^{\prime}(\alpha)+T(\alpha)$ is strictly increasing in $\left(\alpha_{0}, 1\right)$. 


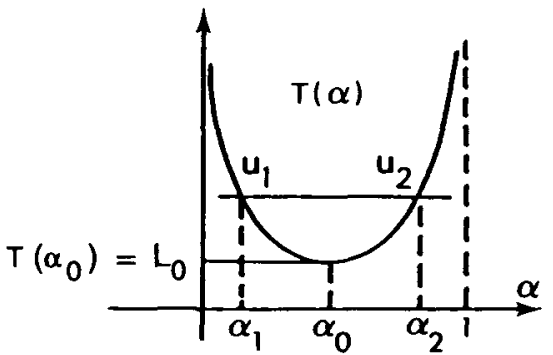

case 1. $f^{\prime}(0)=0$

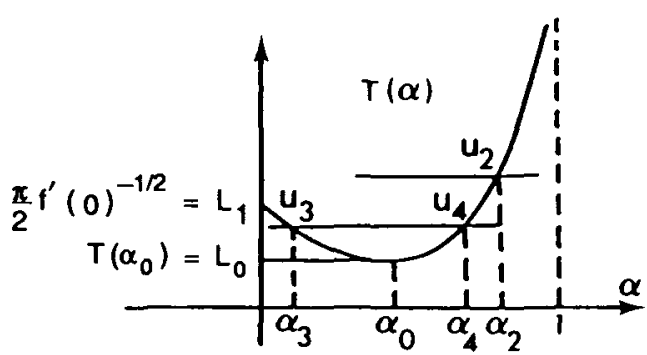

case 2. $f^{\prime}(0)>0$

Figure 2

REMARK 1. In (2.2), we allow $f^{\prime \prime}$ to have infinitely many zeros in $(a, 1)$.

REMARK 2. It follows from the theorem that the time map $T$ has the form given in Figure 2. This means in case $1\left(f^{\prime}(0)=0\right)$ : for short intervals of $L<L_{0}$, there is no positive solution; if $L=L_{0}$, a local bifurcation occurs and we obtain a ("double") nonconstant positive solution; while for $L>L_{0}$, there are precisely two nonconstant positive solutions. In case $2\left(f^{\prime}(0)>0\right)$ : for short intervals of $L<L_{0}$, there is no positive solution; if $L=L_{0}$, a local bifurcation occurs and we obtain a nonconstant positive solution; while for $L_{1}>L>L_{0}$, there are precisely two nonconstant positive solutions; if $L=L_{1}$, a local bifurcation occurs again and we obtain only one positive solution; if $L>L_{1}$, there is exactly one positive solution.

REMARK 3. It can be shown [15] that all the multiple positive solutions of (1.1) satisfying (1.3) obtained in the theorem are ordered; that is, if $u, w$ are two positive solutions of (1.1) satisfying (1.3) with $f$ satisfying (1.2) then $\|u\|_{\infty}<\|w\|_{\infty}$ implies $u(x)<w(x)$ for $x \in(-L, L)$.

REMARK 4. In $(2.2)$, we only allow $f^{\prime \prime}$ to change sign once in $(0,1)$. If $f^{\prime \prime}$ changes sign more than once in $(0,1)$, the time map $T(\alpha)$ could have more than one critical point in $(0,1)$.

EXAMPLE. Choose $f(x)=x^{2}(1-x)$. So $a=1 / 3$. We also note that, after taking one more derivative of $T^{\prime}$ (cf. [12, p. 273]), we compute and find that the integrand of $T^{\prime \prime}(\alpha)$ is always positive for each $\alpha, 0<\alpha<1$, for $f(x)=x^{2}(1-x)$. Thus $T^{\prime \prime}(\alpha)>0,0<\alpha<1$; that is, $T^{\prime \prime}(\alpha)$ is a strictly convex function. This helps us understand the further properties of the time map $T$.

Proposition 1. In case $1\left(f^{\prime}(0)=0\right)$ : we see that if $L>L_{0}$ (see Figure 2), (1.1) has exactly two positive solutions $u_{1}, u_{2}$ with $T^{\prime}\left(\alpha_{1}\right) \neq 0$, $T^{\prime}\left(\alpha_{2}\right) \neq 0$. Then [11, Chapter 24, Theorem A] implies that $u_{1}, u_{2}$ are both nondegenerate in the strong sense that 0 is not in the spectrum of the 
operator $d^{2} / d x^{2}+f(\cdot)$ linearized about either one of $u_{1}, u_{2}$. Further, by Conley index theory [11, Part IV], the Conley indices of $u_{1}$ and $u_{2}$ are

$$
h\left(u_{1}\right)=\Sigma^{1} \text { and } h\left(u_{2}\right)=\Sigma^{0} .
$$

Thus $u_{1}$ is an unstable and $u_{2}$ is a stable solution of the proper initialboundary-value problem for $u_{t}=u_{x x}+f(u)$ (see [11, pp. 530-536] for details). (Note: $\Sigma^{k}(k \geq 0)$ denotes the homotopy type of the pointed $k$-sphere).

Similarly, in case $2\left(f^{\prime}(0)>0\right)$ : if $L>L_{1}$ (see Figure 2$)$, then (1.1) has exactly one positive solution $u_{2}$ with $T^{\prime}\left(\alpha_{2}\right) \neq 0$. Thus $u_{2}$ is nondegenerate. Then $h\left(u_{2}\right)=\Sigma^{0}$; that is, $u_{2}$ is stable. But, if $L_{0}<L<L_{1}$, then (1.1) has exactly two positive solutions $u_{3}, u_{4}, h\left(u_{3}\right)=\Sigma^{1}$ and $h\left(u_{4}\right)=\Sigma^{0}$; that is, $u_{3}$ is unstable and $u_{4}$ is stable.

\section{Proof}

First, through asymptotic expansion of the integrand and direct computation, we have the following propositions.

Proposition 2. Suppose $f$ satisfies (1.2), and let $T$ be defined by (1.9). Then

$$
T(0)=\frac{\pi}{2} f^{\prime}(0)^{-1 / 2} \text { if } f(0)=0 \text { and } f^{\prime}(0)>0,
$$

and

$$
T(0)=\infty \text { if } f(0)=f^{\prime}(0)=0 .
$$

Proposition 3. Suppose $f$ satisfies (1.2), and let $T$ be defined by (1.9). Then $T(1)=\infty$.

We are now in a position to prove the theorem.

Proof of THE THEOREM. The conclusions (2.4) and (2.5) are obtained in Propositions 2 and 3. We now prove (2.3) as follows.

In (1.10), we have

$$
T^{\prime}(\alpha)=2^{-3 / 2} \int_{0}^{\alpha} \frac{\theta(\alpha)-\theta(u)}{(\Delta F)^{3 / 2}} \frac{d u}{\alpha} .
$$

Now, by (1.2), (2.1), (1.11) and (1.12), we get

$$
\begin{gathered}
\theta(0)=0, \\
\theta^{\prime}(0)=0,
\end{gathered}
$$


and

$$
\theta(1)=2 F(1)-f(1)=2 F(1)>0 .
$$

Also by (1.13) and (2.2), we have

$$
\begin{aligned}
& \theta^{\prime \prime}(x)<0 \quad \text { in }(0, a), \\
& \theta^{\prime \prime}(a)=0, \quad \text { and } \theta^{\prime}(a)<0, \\
& \theta^{\prime \prime}(x) \geq 0 \quad \text { in }(a, 1) .
\end{aligned}
$$

So there exists $q, 0<q<1$, the first critical point of $\theta$ in $(0,1)$ such that

$$
\begin{aligned}
\theta(x) & <0 \text { and } \theta^{\prime}(x)<0 \text { in }(0, q), \\
\theta(q) & <0, \text { and } \theta^{\prime}(q)=0, \\
\theta^{\prime}(x) & \geq 0 \text { in }(q, 1) .
\end{aligned}
$$

Hence, by (3.5) and (3.6),

$$
a<q
$$

Thus, by (3.6),

$$
\theta(\alpha)-\theta(u)<0 \quad \text { if } \alpha<q \text { and } u<\alpha .
$$

Hence, for (3.1),

$$
T^{\prime}(\alpha)<0 \quad \text { if } \alpha \leq q .
$$

In addition, $T(1)=\infty$, so $T$ has at least one critical point. Thus, to show $T$ has exactly one critical point, we need only consider $\alpha>q$.

In (1.14), we have

$$
T^{\prime \prime}(\alpha)+\frac{2}{\alpha} T^{\prime}(\alpha)>\frac{2^{-3 / 2}}{\alpha^{2}} \int_{0}^{\alpha}(\Delta F)^{-3 / 2}(\phi(\alpha)-\phi(u)) d u,
$$

where $\phi(x)=x \theta^{\prime}(x)-\theta(x)$. So, by differentiating $\phi$ and (1.13), we get

$$
\phi^{\prime}(x)=x \theta^{\prime \prime}(x)=-x^{2} f^{\prime \prime}(x) \text {. }
$$

Now, by (2.2), (3.5), (3.6), and (3.11), we have $\phi(0)=0, \phi^{\prime}(0)=0$,

$$
\phi(q)=q \theta^{\prime}(q)-\theta(q)=-\theta(q)>0,
$$

and

$$
\begin{aligned}
& \phi^{\prime}(x)<0 \quad \text { in }(0, a), \\
& \phi^{\prime}(a)=0, \\
& \phi^{\prime}(x) \geq 0 \quad \text { in }(a, 1) .
\end{aligned}
$$


So

$$
\begin{array}{ll}
\phi(\alpha)-\phi(u) \geq 0 & \text { if } q<\alpha<1 \text { and } u<\alpha, \\
\phi(\alpha)-\phi(u)>0 & \text { if } q<\alpha<1 \text { and } u<a .
\end{array}
$$

Hence, for (1.14), the integrand is always nonnegative if $0<\alpha<q$ and $0<u<\alpha$ and is always positive if $q<\alpha<1$ and $0<u<a$. Thus

$$
T^{\prime \prime}(\alpha)+\frac{2}{\alpha} T^{\prime}(\alpha)>0 \quad \text { in } q<\alpha<1 .
$$

If $T^{\prime}(\alpha)=0$ for some $\alpha, q<\alpha<1$, then $T^{\prime \prime}(\alpha)>0$. Hence $T$ has exactly one critical point, a minimum at $\alpha_{0}$, for some $\alpha_{0}$ in $(0,1)$. Moreover, by (3.9) and (3.15), it follows that $T$ is strictly decreasing in $\left(0, \alpha_{0}\right)$, and $\alpha T^{\prime}(\alpha)+T(\alpha)$ is strictly increasing in $\left(\alpha_{0}, 1\right)$. This completes the proof of the theorem.

\section{Acknowledgment}

We should like to thank Dr. Y.-H. Wan for many helpful conversations.

\section{References}

[1] A. Ambrosetti, 'On the exact number of positive solutions of convex nonlinear problems,' Bulletino, U.M.I. (5) 15-A (1978), 610-615.

[2] A. Ambrosetti and P. Hess, 'Positive solutions of asymptotically linear elliptic eigenvalue problems,' J. Math. Anal. Appl. 73 (1980), 411-422.

[3] H. Berestycki, 'Le nombre de solutions de certain problèmes semilinéaires elliptiques,' J. Funct. Anal. 40 (1980), 1-29.

[4] M. Bramson, Convergence of solutions of the Kolmogorov equation to travelling waves, Memoirs Amer. Math. Soc. 285, 1983.

[5] E. N. Dancer, 'On the number of positive solutions of weakly non-linear elliptic equations when a parameter is large,' Proc. London Math. Soc. 53 (1987), 429-452.

[6] D. G. de Figueiredo, 'On the uniqueness of positive solutions of the Dirichlet problem $-\Delta u=\lambda \sin u$,' pp. 80-83 in Nonlinear Partial Differential Equations and Applications vol. 7, H. Brezis and J. L. Lions (editors) (Pitman, London, 1984).

[7] I. Gelfand, 'Some problems in the theory of quasilinear equations', Amer. Math. Soc. Translations 29 (1963), 295-381.

[8] A. Kolmogoroff, I. Petrovsky, and N. Piscounoff, 'Étude de l'équations de la diffusion avec croissance de la quantité de matière et son application à un problème biologique', Bull. Univ. Moscow, Ser. Internat., Sec. A 1 (1937), 1-25.

[9] M. B. Sevryuk, Reversible Systems (Lecture Notes in Math. 1211, Springer-Verlag, Berlin, Heidelberg, 1986).

[10] T. I. Seidman, 'Asymptotic growth of solutions of $-\Delta u=\lambda f(u)$ for large $\lambda$,' Indiana Univ. Math. J. 30 (1981), 305-311. 
[11] J. Smoller, Shock Waves and Reaction-Diffusion Equations (Springer-Verlag, New York, 1983).

[12] J. Smoller and A. Wasserman, 'Global bifurcation of steady-state solutions,' J. Differential Equations 39 (1981), 269-290.

[13] J. Smoller and A. Wasserman, 'Generic bifurcation of steady-state solutions,' J. Differential Equations 52 (1984), 432-438.

[14] S.-H. Wang, 'A correction for a paper by J. Smoller and A. Wasserman,' J. Differential Equations 77 (1989), 199-202.

[15] S.-H. Wang and N. D. Kazarinoff, 'Bifurcation of steady-state solutions of a scalar reaction-diffusion equation in one space variable,' J. Austral. Math. Soc. (Series A) 52 (1992), 343-355.

Department of Mathematics

National Tsing Hua University

Hsinchu, Taiwan 300

R.O.C. 